\title{
Research Paper \\ Comparison of Effectiveness Self-care Returns Two Methods of Focus Group Discussions and Teach-back on Lifestyle of Pregnant Women
}

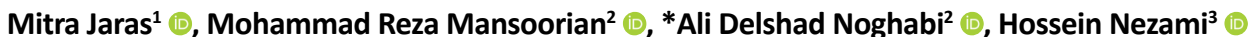

1. Department of Nursing, School of Nursing and Midwifery, Arak University of Medical Sciences, Arak, Iran

2. Department of Community Health Nursing and Nursing Management, School of Nursing, Social Development and Health Promotion Research Center, Gonabad University of Medical Sciences, Gonabad, Iran.

3. Department of Basic Science, School of Medicine, Social Development and Health Promotion Research Center, Gonabad University of Medical Sciences, Gonabad, Iran.

\begin{tabular}{|c|c|}
\hline $\begin{array}{l}\text { Use your device to scan } \\
\text { and read the article online }\end{array}$ & ditation Jaras M, Mansoorian M, Delshad Noghabi A, Nezami H. [Comparison of Effectiveness Self-care Returns Two Methods \\
\hline 口保回 & of Focus Group Discussions and Teach-back on Lifestyle of Pregnant Women (Persian)]. Quarterly of "The Horizon of Medical \\
\hline & Sciences". 2020; 26(1):94-107. https://doi.org/10.32598/hms.26.1.2761 \\
\hline 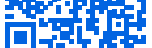 & doi'https://doi.org/10.32598/hms.26.1.2761 \\
\hline
\end{tabular}

(c) (i) (5)

Received: 09 Sep 2018 Accepted: 13 Oct 2019 Available Online: 01 Jan 2020

Key words: Self-care education Focus group discussion, Teach-back, Lifestyle

\section{ABSTRACT}

Aims This study aimed to compare the effects of self-care education using focus group discussion and teachback method on the lifestyle promotion of pregnant women.

Methods \& Materials In this randomized field trial, participants were 90 pregnant women referred to the community health centers in Gonabad, Iran with no history of physical and psychological problems that can affect their self-care. They were randomly selected from among the three health centers and divided into three groups of focus group discussion, teach-back, and control. The data collection tools were a demographic form, and Walker's Health-Promoting Lifestyle Profile (HPLP) questionnaire completed before and one month after the intervention. The collected data were analyzed in SPSS v. 20 software using independent t-test, paired ttest and ANOVA. The P-value $<0.05$ was set as the significance level.

Findings There was no significant difference between the three groups in terms of educational level $(P=0.029)$, occupation ( $P=0.090)$, income $(P=0.099)$, and gestational age $(P=0.741)$, and they were homogeneous. After intervention, all aspects of health-promoting lifestyle (except stress management) in two groups of teach-back $(P<0.001)$ and focus group discussion $(P<0.001)$ were significantly improved compared to the control group $(P=0.66)$. In comparing the two educational methods, focus group discussion had a more significant impact on the lifestyle than the teach-back method.

Conclusion Focus group discussion method is recommended for the self-care education of pregnant women.

\section{Extended Abstract}

\section{Introduction}

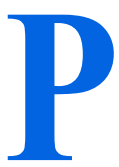

regnancy is a crucial stage in women's lives and due to the direct effect of maternal health on fetal health, the importance of this period is much more significant. Given the role of the health care team in improving health and teaching self-care behaviors, it is possible to identify these behaviors by planning interventions and improve the level of self-care and thus the lifestyle of pregnant women. This study aimed to compare the effects of selfcare education given by focus group discussion and teach-back methods on the lifestyle of pregnant women.

\section{* Corresponding Author:}

Ali Delshad Noghabi

Address: Department of Community Health Nursing and Nursing Management, School of Nursing, Social Development and Health Promotion Research Center, Gonabad University of Medical Sciences, Gonabad, Iran.

Tel: +98 (51) 57220576

E-mail: ali_delshad2000@yahoo.com 
In a study examined the effect of self care education based on teach-back method (4 sessions) on the promotion of self care behaviors in 70 patients with type 2 diabetes in Hamedan, Iran in two test and control groups. Their study showed a significant improvement in self-care behaviors of patients in test group after intervention compared to the controls. They concluded that teach-back method can have an important role in reducing the disease and improving the life quality of patients [1]. In other research the effect of educational booklet evaluated using focus group discussion method on 56 primary school students' knowledge of waste pollution. Students were divided into 7 groups and were assessed before and after intervention. They reported that focus group discussion could improve the students' knowledge of waste pollution [2].

\section{Materials and Methods}

This is a randomized field Trial. Participants were 90 pregnant women referred to the community health centers in Gonabad, Iran with no history of physical and psychological problems that can affect their selfcare. They were randomly selected from among the three health centers and divided into three groups of focus group discussion, teach-back, and control. The first two groups received self-care education about pre- natal care using focus group discussion and teach-back method, while the control group received a routine education. The data collection tools were a demographic form, and Walker's Health-Promoting Lifestyle Profile (HPLP) questionnaire completed before and one month after the intervention.

\section{Results}

There was no significant difference between the three groups in terms of educational level $(\mathrm{P}=0.029)$, occupation $(\mathrm{P}=0.090)$, income $(\mathrm{P}=0.099)$, and gestational age $(\mathrm{P}=0.741)$, and they were homogeneous (Table 1). After intervention, health responsibility, spiritual growth, physical activity, interpersonal relationships, and nutrition (aspects of health-promoting lifestyle) of teach-back $(\mathrm{P}<0.001)$ and focus group discussion $(\mathrm{P}<0.001)$ groups were significantly improved compared to the control group $(\mathrm{P}=0.66)$ except in stress management dimension which showed no significant difference (Table 2). In comparing the effects of two educational methods, focus group discussion had a more significant impact on the lifestyle of pregnant women compared to the teach-back method (Table 3).

Table 1. Frequency distribution of demographic variables in the study groups

\begin{tabular}{|c|c|c|c|c|c|}
\hline & & \multicolumn{3}{|c|}{ Groups } & \multirow{3}{*}{$\mathrm{X} 2(\mathrm{P})$} \\
\hline & \multirow[t]{2}{*}{ Characteristics } & \multicolumn{3}{|c|}{ No. (\%) } & \\
\hline & & Teach-Back & Focus Group Discussion & Control & \\
\hline \multirow{3}{*}{ Education } & Lower than high school & $3(10)$ & $3(10)$ & $3(10)$ & \multirow{3}{*}{$0.333(0.259)$} \\
\hline & High school diploma & $8(26.66)$ & $7(23.33)$ & $7(23.33)$ & \\
\hline & University degree & 19(63.33) & $20(66.66)$ & $20(66.66)$ & \\
\hline \multirow{2}{*}{ Occupation } & Employed & $10(33.3)$ & $7(23.3)$ & 13(43.3) & \multirow{2}{*}{$2.70(0.906)$} \\
\hline & Housekeeper & $20(66.66)$ & $23(76.66)$ & & \\
\hline \multirow{3}{*}{ Income } & Sufficient & 28(93.33) & $27(90)$ & $27(100)$ & \multirow{3}{*}{$1.36(0.998)$} \\
\hline & Less than sufficient & $1(3.33)$ & $2(6.66)$ & $1(3.33)$ & \\
\hline & More than sufficient & $1(3.33)$ & $1(3.33)$ & $2(6.66)$ & \\
\hline \multirow{3}{*}{ Gestational age } & $<12$ weeks & $3(10)$ & $4(13.33)$ & $3(10)$ & \multirow{3}{*}{$1.267(0.741)$} \\
\hline & 13-24 weeks & $17(56.66)$ & $17(56.66)$ & $16(53.33)$ & \\
\hline & $>24$ weeks & $10(33.33)$ & $9(30)$ & $11(36.66)$ & \\
\hline
\end{tabular}


Table 2. Aspects of lifestyle in the study groups pre and post intervention

\begin{tabular}{|c|c|c|c|c|c|}
\hline \multirow{2}{*}{\multicolumn{2}{|c|}{ Health-Promoting Lifestyle Dimensions }} & \multicolumn{3}{|c|}{ Mean \pm SD } & \multirow{2}{*}{$\mathbf{P}$} \\
\hline & & Teach-Back & Focus Group Discussion & Control & \\
\hline \multirow{2}{*}{ Spiritual growth } & Pre-test & $25.4 \pm 5.11$ & $26.83 \pm 5.47$ & $25.66 \pm 5.13$ & 0.533 \\
\hline & post-test & $27.53 \pm 4.9$ & $30.33 \pm 4.27$ & $25.73 \pm 5.08$ & 0.003 \\
\hline \multirow{2}{*}{ Health responsibility } & Pre-test & $24.33 \pm 4.88$ & $26.53 \pm 4.41$ & $25.6 \pm 3.72$ & 0.153 \\
\hline & post-test & $27.03 \pm 4.62$ & $30.10 \pm 3.12$ & $25.73 \pm 3.80$ & $<0.001$ \\
\hline \multirow{2}{*}{ Interpersonal relationships } & Pre-test & $25.8 \pm 5.3$ & $26.23 \pm 3.97$ & $25.1 \pm 4.16$ & $P=0.62$ \\
\hline & post-test & $27.53 \pm 5.34$ & $29.33 \pm 4.04$ & $25.1 \pm 3.99$ & $P=0.00$ \\
\hline \multirow{2}{*}{ Physical activity } & Pre-test & $15.36 \pm 4.77$ & $16.46 \pm 3.8$ & $16.3 \pm 4.82$ & 0.59 \\
\hline & post-test & $18.5 \pm 5.22$ & $20.66 \pm 4.51$ & $16.46 \pm 4.86$ & 0.005 \\
\hline \multirow{2}{*}{ Nutrition } & Pre-test & $25.9 \pm 5.08$ & $27.13 \pm 4.37$ & $26.46 \pm 4.96$ & 0.61 \\
\hline & post-test & $27.93 \pm 4.92$ & $29.63 \pm 3.5$ & $26.56 \pm 4.87$ & 0.057 \\
\hline \multirow{2}{*}{ Stress management } & Pre-test & $22.53 \pm 4.89$ & $22.55 \pm 4.67$ & $25.33 \pm 4.16$ & 0.259 \\
\hline & post-test & $22.7 \pm 4.77$ & $22.7 \pm 4.74$ & $25.43 \pm 4.17$ & 0.269 \\
\hline
\end{tabular}

Table 3. Mean \pm SD of lifestyle score in the study groups pre and post intervention

\begin{tabular}{|c|c|c|c|}
\hline \multirow{2}{*}{ Group } & \multicolumn{2}{|c|}{ Mean \pm SD } & \multirow{2}{*}{ t-test } \\
\hline & Pre-test & Post-test & \\
\hline \multirow{2}{*}{ Teach-back } & \multirow{2}{*}{$135.46 \pm 21.99$} & \multirow{2}{*}{$147.20 \pm 2.58$} & $t=-5.23$ \\
\hline & & & $P<0.001$ \\
\hline \multirow{2}{*}{ Focus group discussion } & \multirow{2}{*}{$143.46 \pm 17.21$} & \multirow{2}{*}{$160.50 \pm 16.36$} & $t=-10.16$ \\
\hline & & & $P<0.001$ \\
\hline \multirow{2}{*}{ Control } & \multirow{2}{*}{$138.43 \pm 20.28$} & \multirow{2}{*}{$138.90 \pm 19.95$} & $t=-2.14$ \\
\hline & & & $\mathrm{P}=0.04$ \\
\hline \multirow{2}{*}{ ANOVA results } & $F=1.23$ & $F=9.08$ & \\
\hline & $P=0.29$ & $P<0.001$ & \\
\hline
\end{tabular}

\section{Conclusion}

To our knowledge, there is no randomized field trial that compares the effects of self-care education using focus group discussion and teach-back method on the lifestyle of pregnant women. Based on the results, it is recommended to provide self-care education for pregnant women during pregnancy using focus group discussion method.

\section{Ethical Considerations}

\section{Compliance with ethical guidelines}

This study has ethical approval with code: IR.GMU. REC.1396.104

\section{Funding}

This study was extracted from a master thesis, and received financial support from the Deputy for Research and Technology Gonabad University of Medical Sciences (Code: 96/148).

\section{Authors' contributions}

Designing the study, Manuscript preparation: Mitra Jaras, Ali Delshad Noghabi, Mohammad Reza Mansoorian; Data analysis, data collection: Mitra Jaras, Hossein Nezami; Reading and approving the final version: All authors 


\section{Conflicts of interest}

The authors declare no conflict of interest

Acknowledgements

The authors would like to thank the Deputy for Research and Technology and the School of Nursing of Gonabad University of Medical Sciences as well as all pregnant women for their support and cooperation. Teaching through focus group discussion increases the level of self-care in pregnant women due to more interaction and communication, and the use of each other's experiences. 
This Page Intentionally Left Blank 


\title{
مقايسه اثربخشى آموزش خودمراقبتى به دو روش بحث تَروهى متمركز و آموزش بركثتى بر سبك زندگى زنان باردار
}

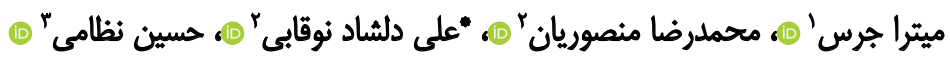 \\ 1. ا.كروه، يرستارى، دانشكده يرستارى و مامايى، دانشكاه علوميزشكى اراك، اراك، ايران.

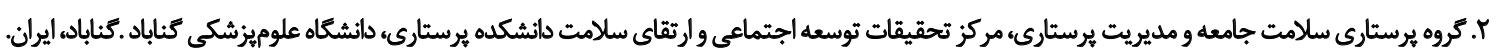

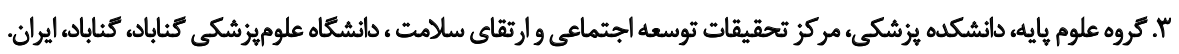

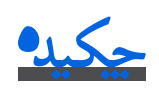

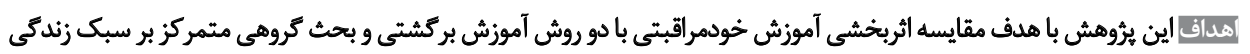
زنان باردار انجام شده است.

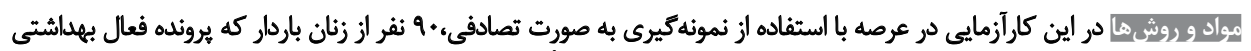

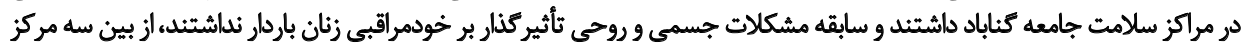

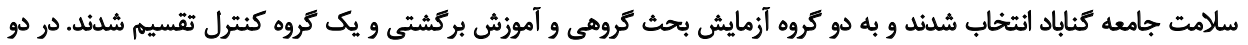

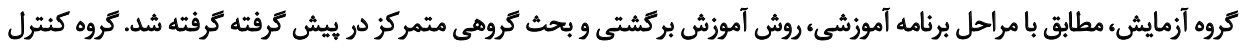

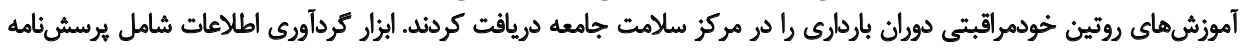

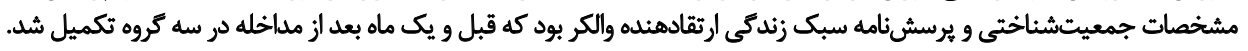

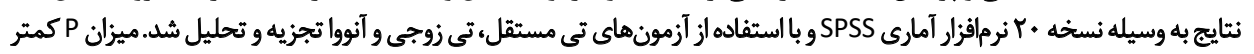

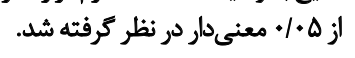

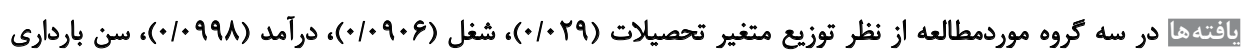

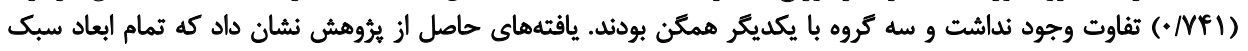

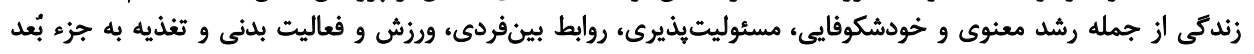

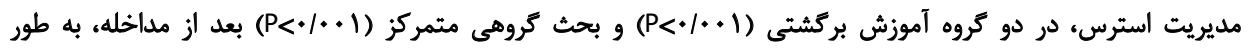

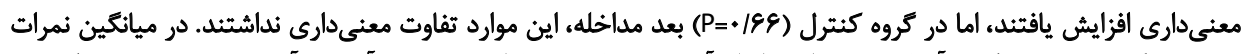

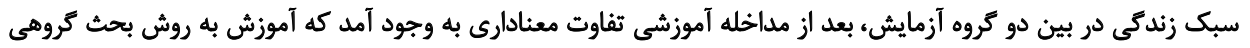

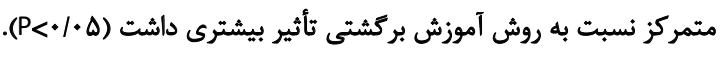

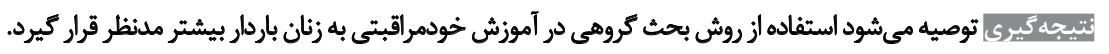

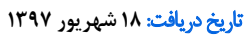

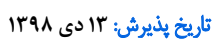

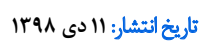

خطرات بالقوه دوران باردارى و كمك به زنان براى اصلاح عوامل

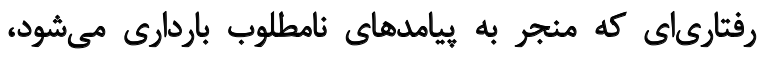

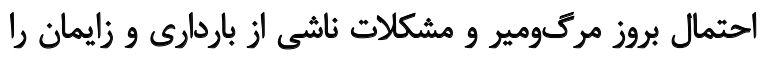

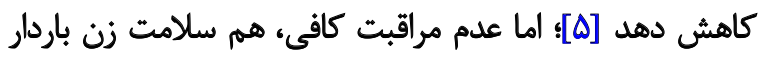

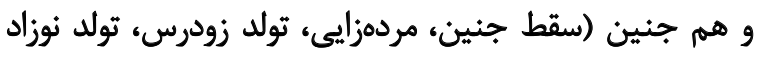

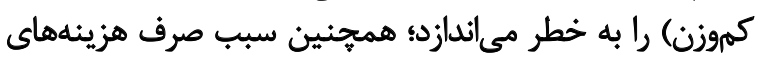

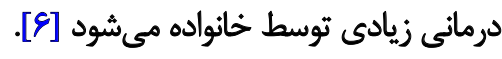

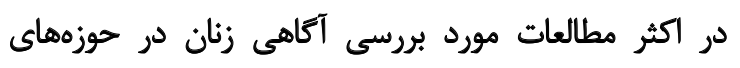

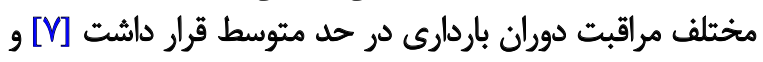

daleo

واردارى مرحلهاى بسيار مهم و حساس در زئدكى زنان است

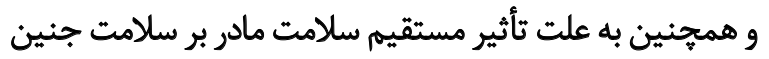

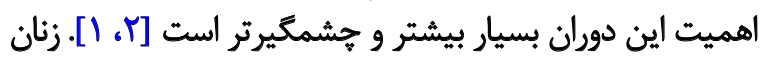

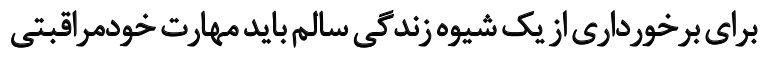

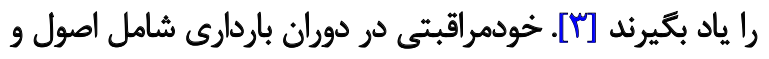

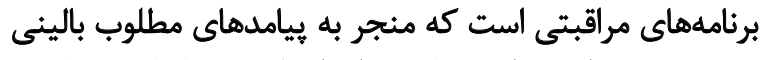

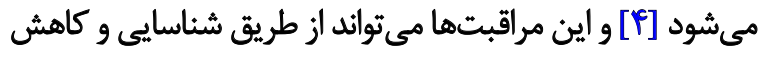

آموزش خودمراقبتى،

روش بحث كروهى بردي متمركز، روش آموزش برثي

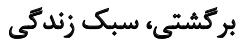

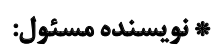

على دلشاد نوقابي منئ

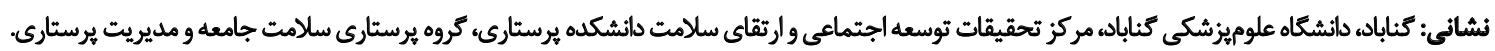
+ تلفن: ali_delshad2000@yahoo.com : بست الكترونيكى • 
شركتكنيدكان توصيه كردهاند [ [T]]. همجنين طبق تحقيقات، يكى از مؤثرترين روشها جهت

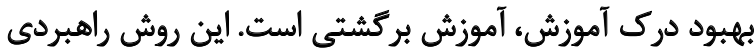

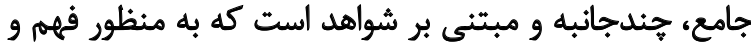

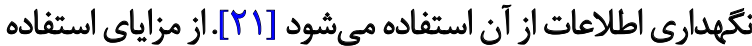

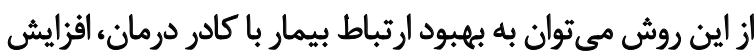

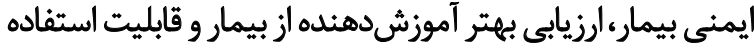

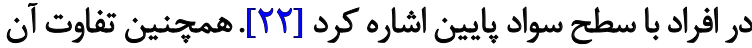

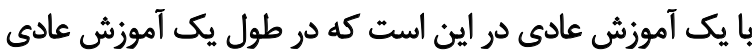

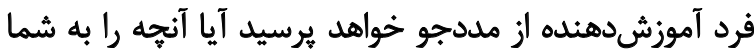

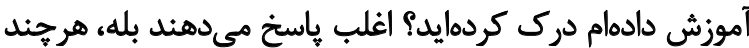

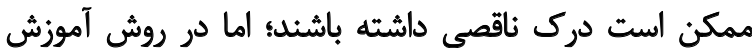

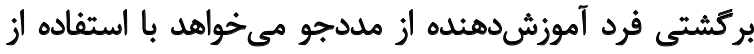

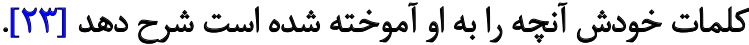

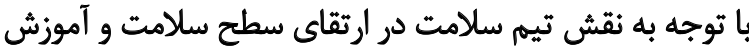

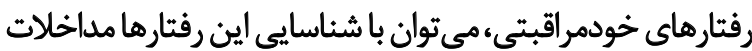

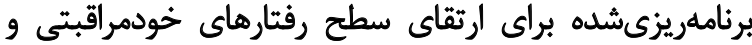

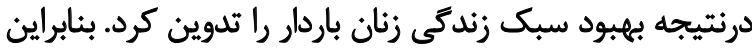

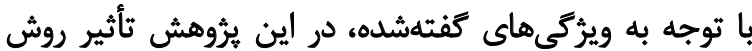

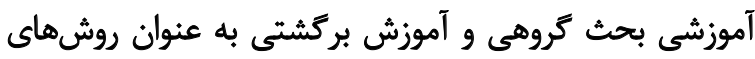

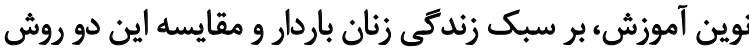

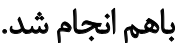

\section{مواد و روشها}

يثروش حاضر از نوع كارآزمايي در عرصه است. جامعه آمارى

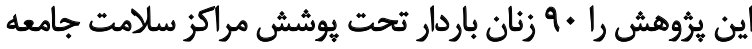

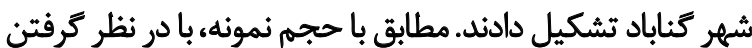

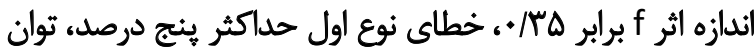

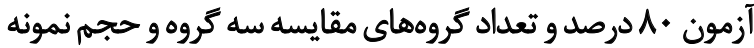

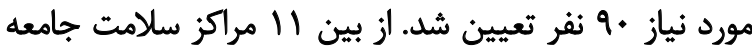

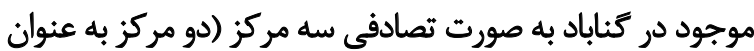

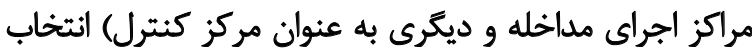

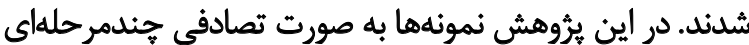

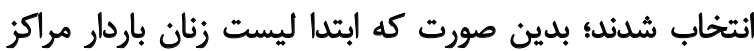

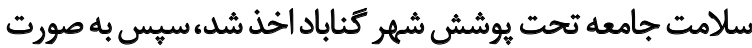

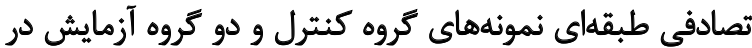

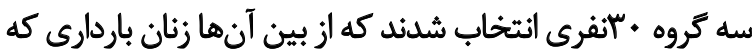

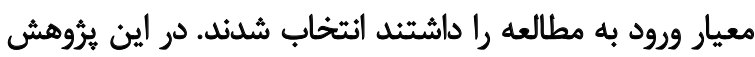

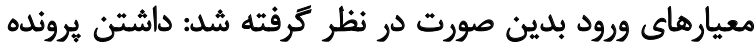

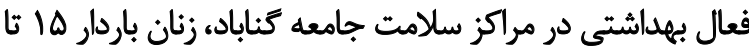

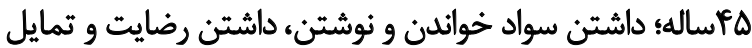

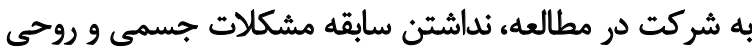
مهم تأثير كذار بر خودمراقبتى (مانند سابقه افسردكى).
در مطالعه درخشان، آكاهي زنان باردار از مسموميت حاملكي در

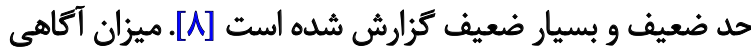

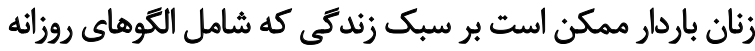

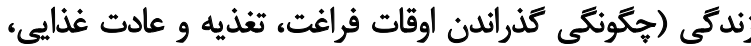

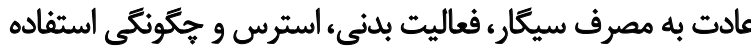

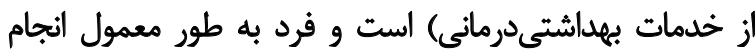

مى دهد، تأثير بحذارد [9]

به نظر ميرسد انتخاب سبك زندكى در باردارى نيز مي تواند

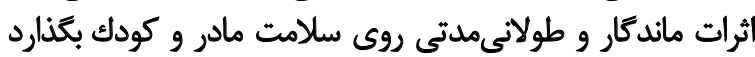

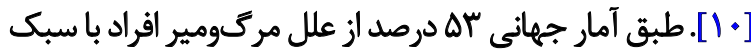

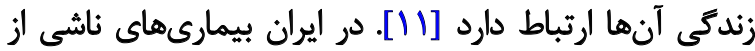

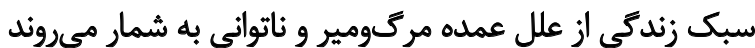

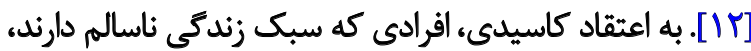

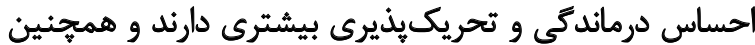

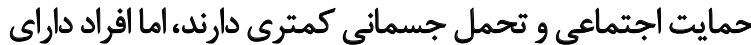

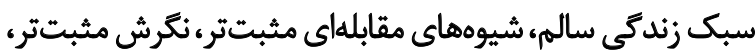

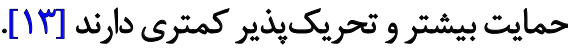

آموزش مؤثر در دوران باردارى در كاهش بيمارىها، عوارض بارض بارد

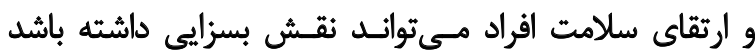

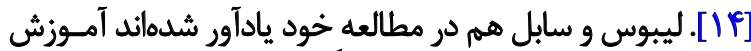

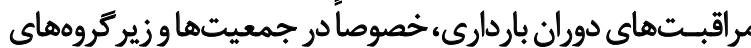

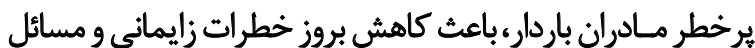

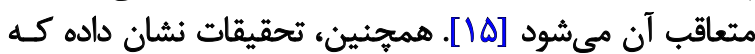

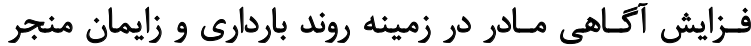

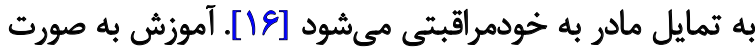

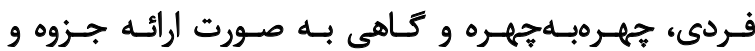

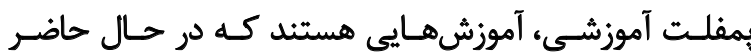

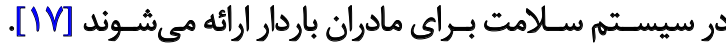
در كشور ما برنامههاي آموزشى در بسيارى از موارد بدون

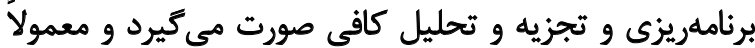

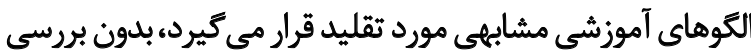

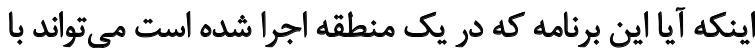

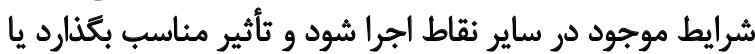

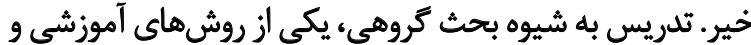

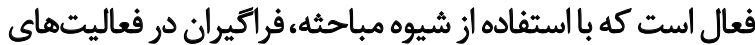

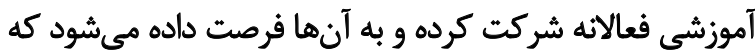

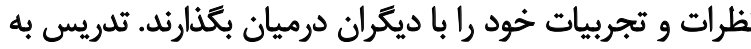

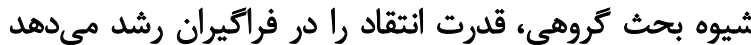

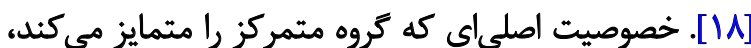

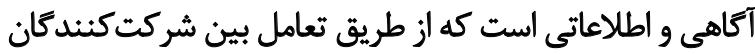

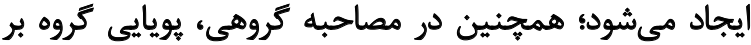

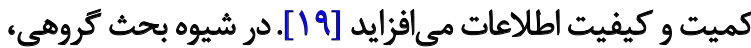

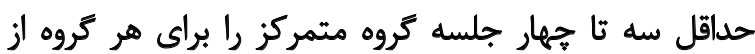


را تكميل كردند. جهت جمعآورى دادهها از فرم مشخصات

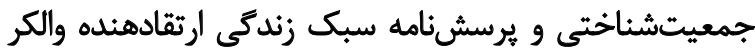

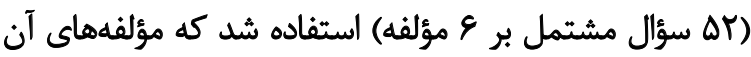

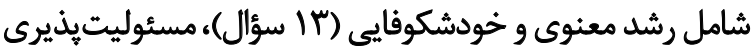

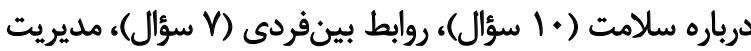

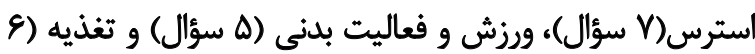

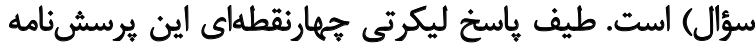

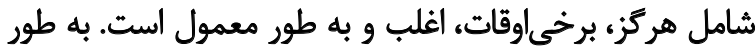

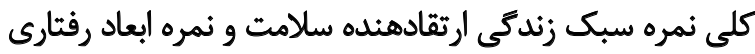

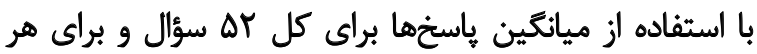

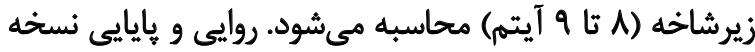

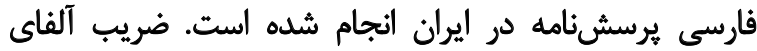

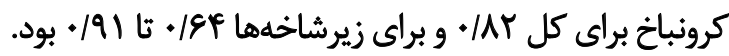

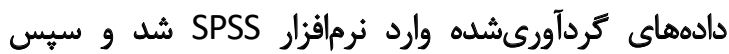

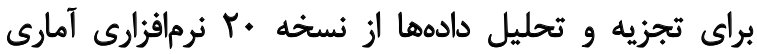

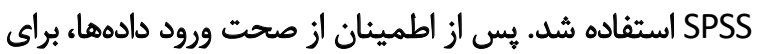

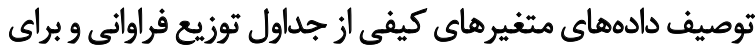

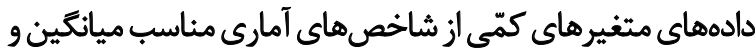

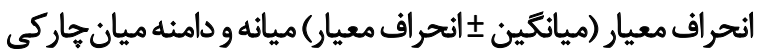

استفاده شده است.

يافتها

بر اساس جدول شماره ا تفاوت معنى دارى از نظر توزيع متغير

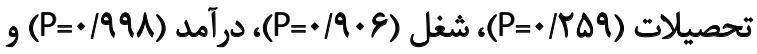

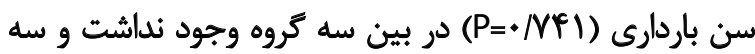
كروه با يكديكر همكن بودند.

جدول شماره Y نشان داد كه قبل از مداخله آموزشى تففاوت

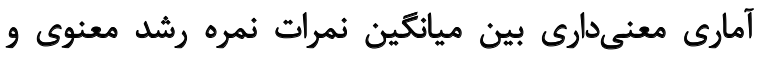

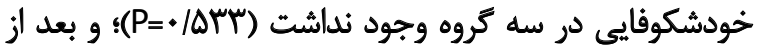

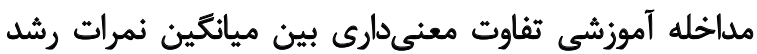

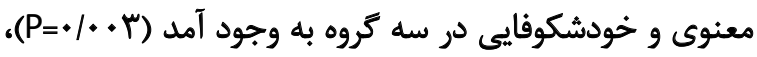

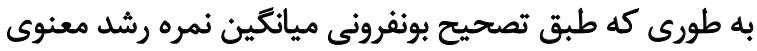

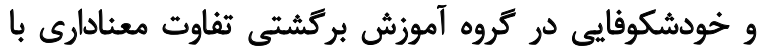

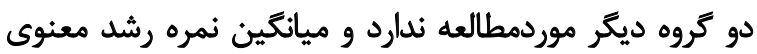

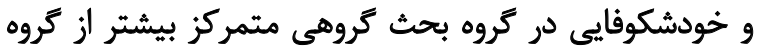

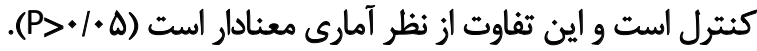

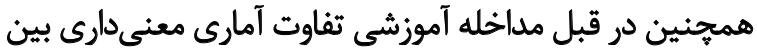

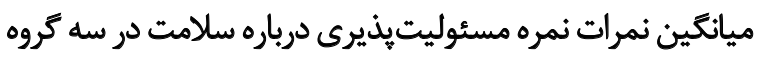

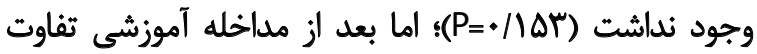

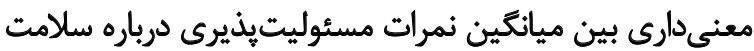

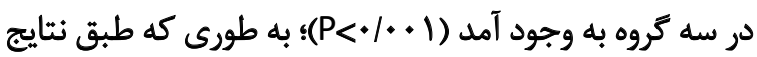

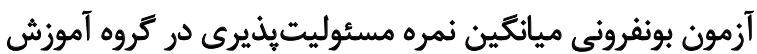
بركشتى تفاوت معنادارى با تروه كنترل نداشت و ميانكين نمره
در كروه آزمايش به روش آموزش بركشتى، ·ب نفر از زنان

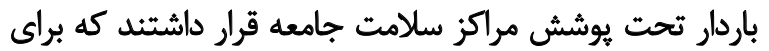

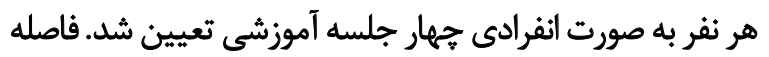

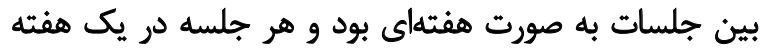

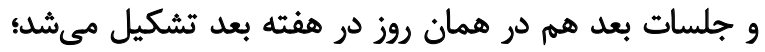

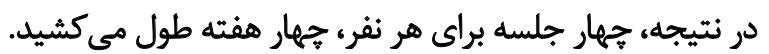

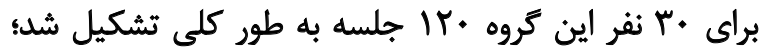

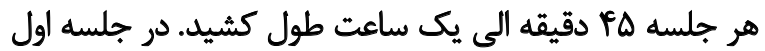

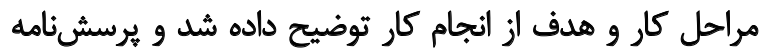

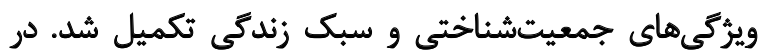

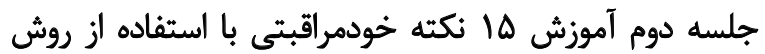

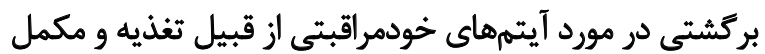

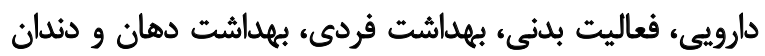

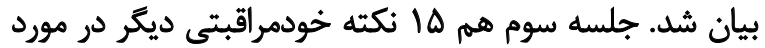

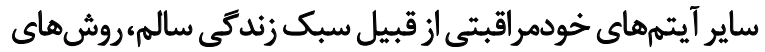

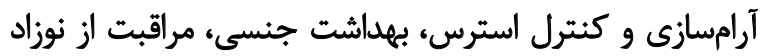

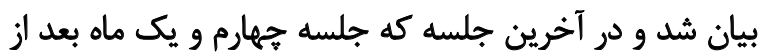

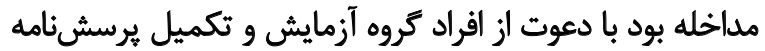

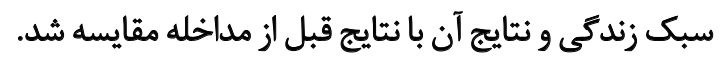

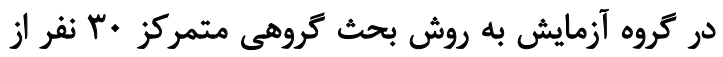

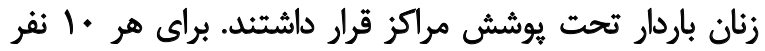

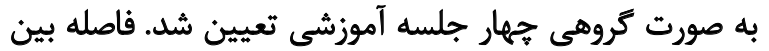

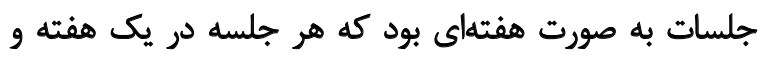

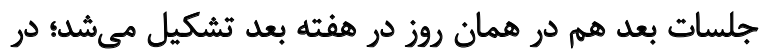

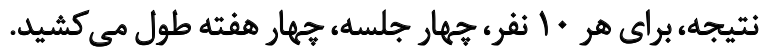

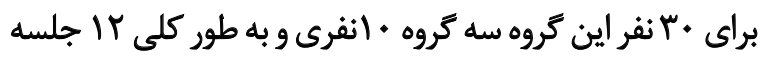

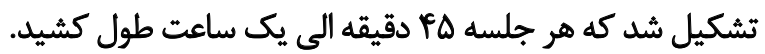
در جلسه اول مراحل كار و هدف از انجام كار توضيح داده شئ شدئ وندي

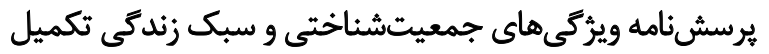

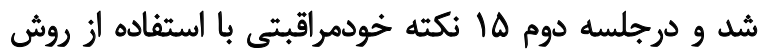

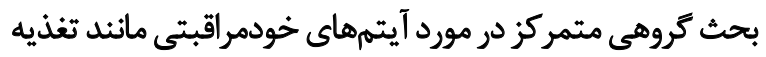

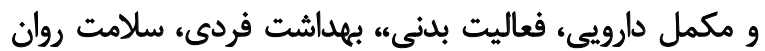

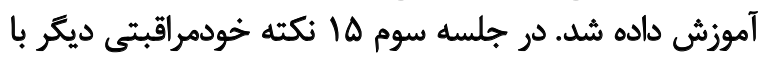

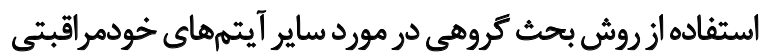

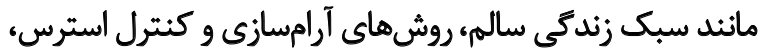

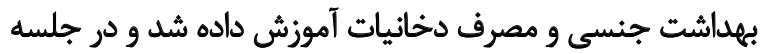

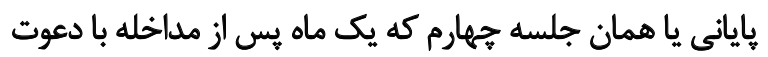

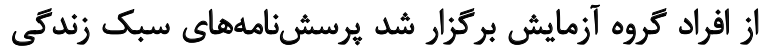

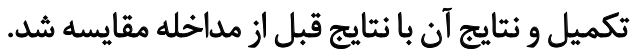

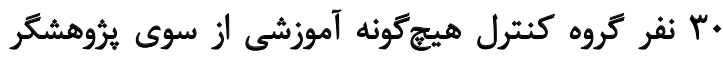

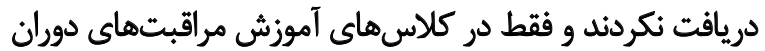

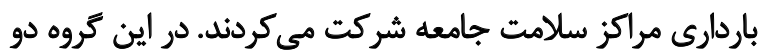

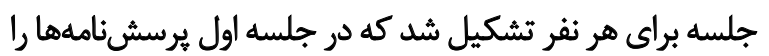

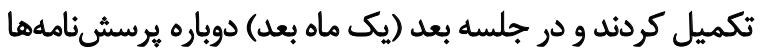


جدول ا.توزيع فراوانى متغيرهاي جمعيتشُناختى در كروهاي موردمطالعه

\begin{tabular}{|c|c|c|c|c|c|}
\hline \multirow{3}{*}{$\begin{array}{c}\text { أزمون كاي اسكوئر } \\
\text { (P-X2) }\end{array}$} & \multicolumn{3}{|c|}{ كروه } & \multirow{3}{*}{\multicolumn{2}{|c|}{ متغيرها }} \\
\hline & كتترل & بحث تروهى متمركز & آموزش بركمشتى & & \\
\hline & \multicolumn{3}{|c|}{ تعداد (درصد) } & & \\
\hline \multirow{3}{*}{$(+/ T \Delta Q)+/ T H T$} & $r(1 \cdot)$ & $r(1 \cdot)$ & $r(1 \cdot)$ & زير دييلم & \multirow{3}{*}{ تحصيلات } \\
\hline & $V(\pi / M T)$ & $V(M / M)$ & $\Lambda($ ME/FG) & دييلم & \\
\hline & $r+(89189)$ & $r \cdot(89 / 88)$ & $19(8 \pi / / 74)$ & دانشكاهي & \\
\hline \multirow{2}{*}{$(. / 9.9) T / N$} & $\mathbb{1 r}(\mathrm{fr} / \mathrm{T})$ & $V(T / M)$ & 1. $(M / M)$ & ش اغل & \multirow{2}{*}{ | شغل } \\
\hline & $\operatorname{IV}(\Delta \& \mid \& \theta)$ & $M(N G / F E)$ & $r \cdot(89188)$ & خانهدار & \\
\hline \multirow{3}{*}{$(+/ 94) \backslash / \% 8$} & $r(1 \cdot \cdot)$ & $r(q \cdot)$ & $r \Lambda(q T / M T)$ & در حد كفاف & \multirow{3}{*}{ درآمد } \\
\hline & $1\left(\pi / \pi^{\prime}\right)$ & $r(8 / 89)$ & $1\left(N / \mu^{\prime}\right)$ & كمتر از حل كفاف & \\
\hline & $r(8 / 89)$ & $1(r / \mu)$ & $1(r / N r)$ & بيشتر از حد كفاف & \\
\hline \multirow{3}{*}{$\left.\left(+/ M^{+}\right)\right)$V/TEV } & $r(1 \cdot)$ & $f(M / T H)$ & $r(1 \cdot)$ & كمتر از rا هفته & \multirow{3}{*}{ سن باردارى } \\
\hline & $18(\Delta r / M T)$ & IV $(\Delta F / \& E)$ & IV (AF/Eg) & 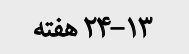 & \\
\hline & $11(m e / F=9)$ & $9(\mu)$ & 1. (MT/M & بيشتر از بم هفته & \\
\hline
\end{tabular}

إن

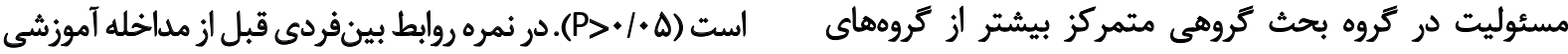

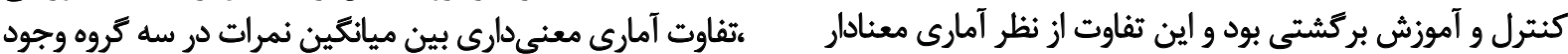

جدول ؟. مؤلفههاي سبك زندكى در كروههاى موردمطالعه در قبل و بعد از مداخله

\begin{tabular}{|c|c|c|c|c|c|}
\hline \multirow{3}{*}{ نتايج آزمون } & \multicolumn{3}{|c|}{ ميانكينثلانحراف معيار } & \multirow{2}{*}{\multicolumn{2}{|c|}{ متغير }} \\
\hline & \multirow{2}{*}{ كروه كتترل } & \multirow{2}{*}{ كروه أزمون بحث كروهى متمركز } & \multirow{2}{*}{ 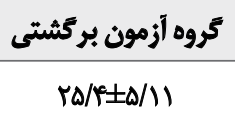 } & & \\
\hline & & & & 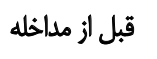 & رشد معنوى و \\
\hline $\mathrm{P}=\star / \bullet r$ & $r \Delta / N^{\prime \prime} \pm \Delta / \bullet \Lambda$ & $r \cdot / r \Psi \pm / r V$ & $r V / \Delta r \pm P / 9$ & بعل از مداخله & خودشكوفايي \\
\hline $\mathrm{P}=\cdot / \Delta \Delta H^{\prime}$ & 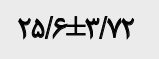 & $r q / \Delta r \pm F / F)$ & $r F / M T+F / M$ & 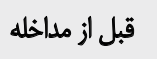 & هسئوليت يذيرى درباره \\
\hline$P<+/ *+1$ & $r \Delta / \mu^{\prime} \pm r / \Lambda$ & $r \cdot / 1 \cdot \pm r / 1 r$ & $r V / \cdot r \pm f / g r$ & 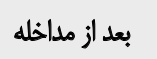 & سلامت \\
\hline $\mathrm{P}=\cdot|g \pi|$ & $r \Delta / \backslash \pm \Gamma / \backslash \&$ & $r q / R r \pm r / q V$ & $r \Delta / \Lambda \pm \Delta / \mu$ & 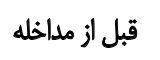 & \\
\hline$P=\bullet / \bullet+r$ & $r \Delta / \triangle \pm r / q q$ & $r q / \pi r \pm f / * f$ & $r V / \Delta H \pm \Delta / M F$ & بعد ازز مداخله & روابط بينفردى \\
\hline$P=.109$ & $18 / R \pm F / A T$ & $18 / F \pm \Psi / A$ & $10 /$ reIfIW & قبل از مداخله & \\
\hline $\mathrm{P}=\bullet / \bullet \bullet$ & IE/FEIF/A & $Y \cdot \mid g g \pm r(\Delta)$ & $W \Delta \pm \Delta / Y Y$ & بعدل از مداخله & ورزش و فعاليت بلنى \\
\hline$P=.|8|$ & re/egtr/as & $r V / I r \pm T / M V$ & $r \Delta / q \pm \Delta / \cdot A$ & قبل از مداخله & تخذه \\
\hline $\mathrm{P}=\cdot / \cdot \Delta \mathrm{V}$ & YQ/OSIF/AV & $r q / T^{\prime} \pm$ T/ब & TW/AYIF/9Y & بعد از مداخله & مأي \\
\hline $\mathrm{P}=\cdot / T \Delta q$ & $r \Delta / M \pm F / M$ & Tr/ADEF/RV & $r r / \Delta r \pm r / 1 q$ & 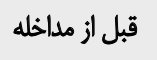 & \\
\hline $\mathrm{P}=. /$ Yeq & $r \Delta / T T \pm F / I V$ & rr/GVIFM & $r r / N \pm P / W$ & بعد از مداخله & \\
\hline
\end{tabular}


جدول با. ميانكين و انحراف معيار نمره سبك زندكى در كروههاى موردمطالعه قبل و بعد از مداخله

\begin{tabular}{|c|c|c|c|}
\hline \multirow{2}{*}{$\begin{array}{c}\text { آماره آزمون } \\
\text { P }\end{array}$} & بعد از مداخله & ق قبل از مداخله & \multirow{2}{*}{ كروه } \\
\hline & \multicolumn{2}{|c|}{ مياتكين لانحراف معيار } & \\
\hline $\begin{array}{l}t=-\Delta / \pi T^{+} \\
P<. / . .1\end{array}$ & $I F V / r+ \pm T / Q A$ & 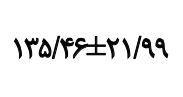 & أموزش بركشتى \\
\hline $\begin{array}{c}\mathrm{t}=-1 . / 1 \varepsilon^{+} \\
\mathrm{P}<* / \ldots 1\end{array}$ & $18 \cdot / 0 \cdot \pm 18 / r \varepsilon$ & $|F T / e \& \pm| V / K \mid$ & بحق كروهى متمركز \\
\hline \multirow[t]{3}{*}{$\begin{array}{c}t=-r / N f^{+} \\
P=+1+f\end{array}$} & $11 \% N q \cdot \pm 19 / 9 \Delta$ & IrN/TETH=/MA & كنترل \\
\hline & $F=q /+1^{*}$ & $F=V / T r$ & آماره آزمون \\
\hline & $P<+1 .+1$ & $\mathrm{P}=. / \mathrm{T}$ & $\mathbf{P}$ \\
\hline
\end{tabular}

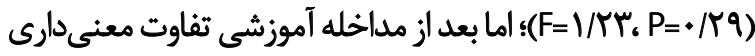

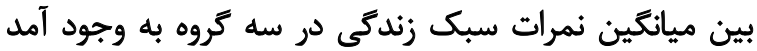

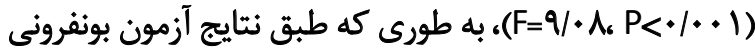

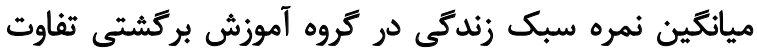

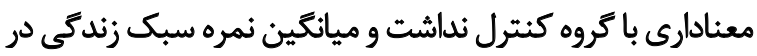

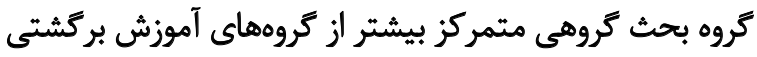

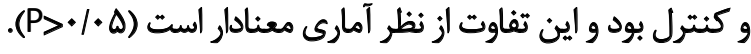

نتايج آزمون تي زوجى نشان داد كه بعد از مداخله، ميانكين

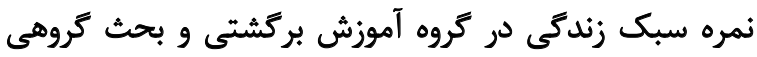

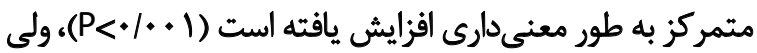

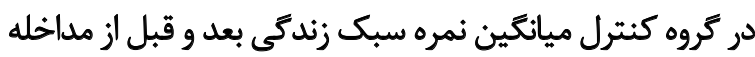
آموزشى تفاوت آمارى معنى كئردارى باهم نداشتند.

As

در اين مطالعه به مقايسه اثربخشى آموزش خودمراقبتى به دو

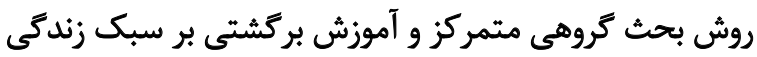

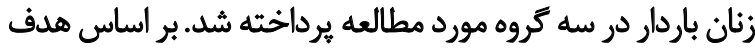

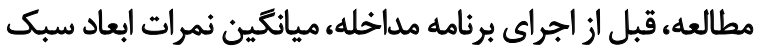

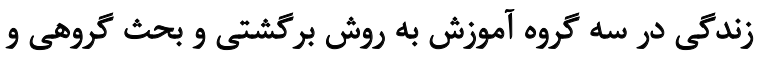

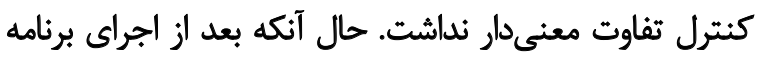

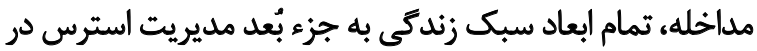

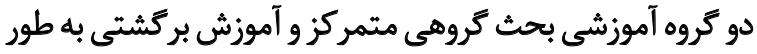

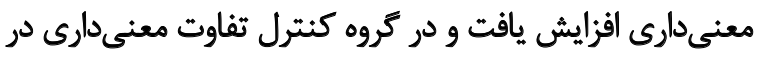
قبل و بعد از مداخله وجود نداشت. در مطالعه راوسج وارئ و همكاران

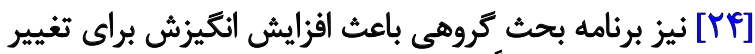

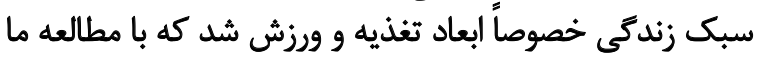

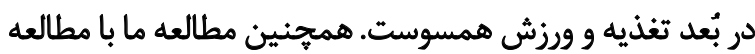

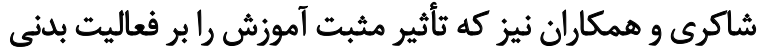

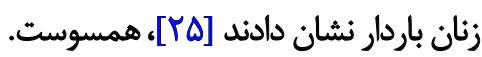

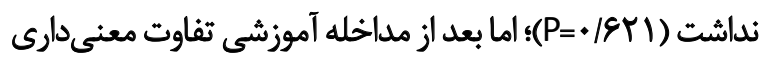

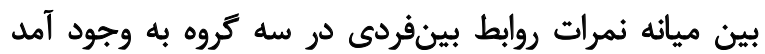

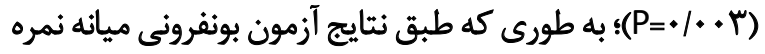

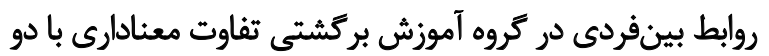

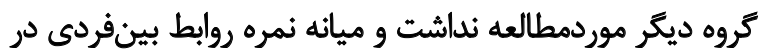

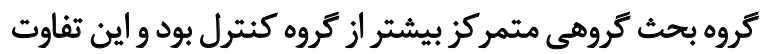
از نظر آمارى معنادار است (ه) (P> (P)

قبل از مداخله آموزشي تفاوت آمارى معنى دارى بين ميانگينين

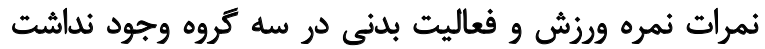

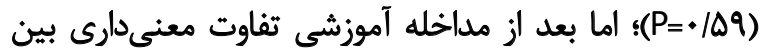

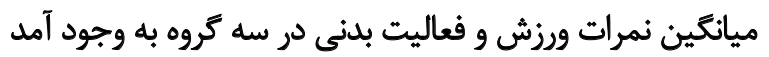

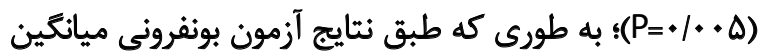

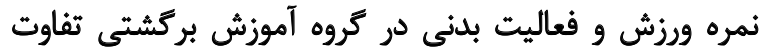

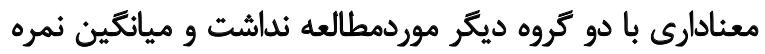

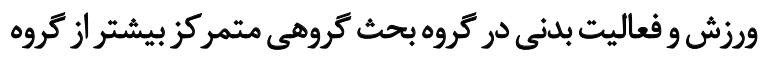

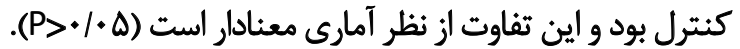
در نمره تغذيه قبل از مداخله آموزشى، تفاوت آمارى معنى دارى

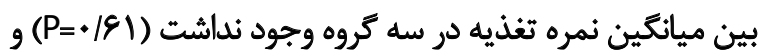

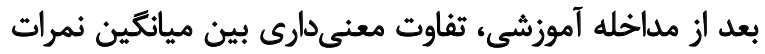
تغذيه در سه

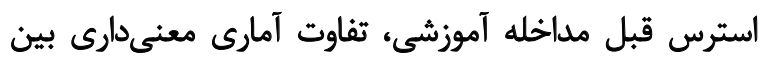

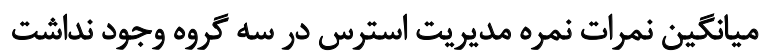

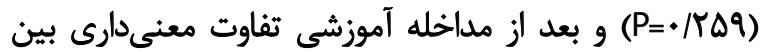

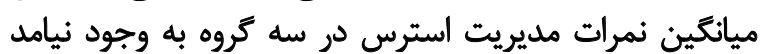
(P=*/T\&q)

طبق جدول شماره ب، آزمون آناليز واريانس يكسطرفه نشان

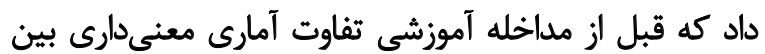

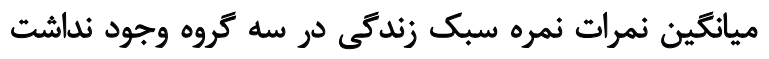


با خود قرار گيرند و امكان مقايسه و تجديد نظر در رفتار و نكرش

خود را بيابند [آب].

در روش بحث تروهى قدرت بيان فراكير تقويت شده و

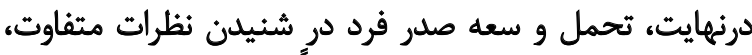

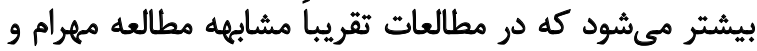

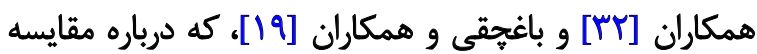
دو روش بحث كروهى و سخنرانى است مؤيد اين مطلب است

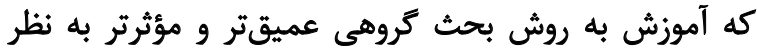

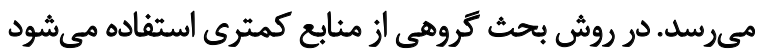

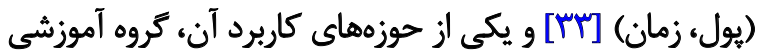

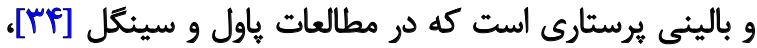

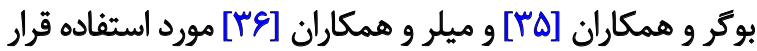

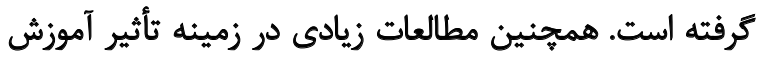

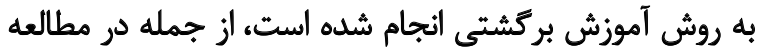

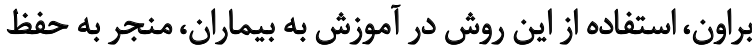

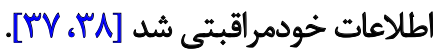

\section{تتيجلمكيرى}

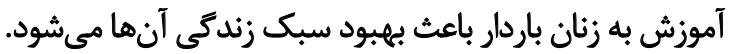

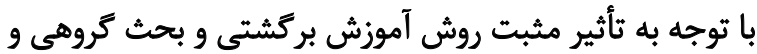

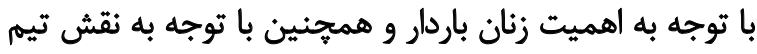

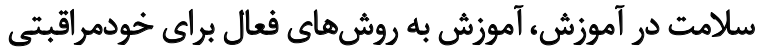

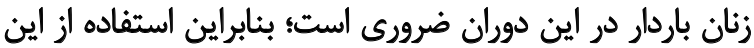

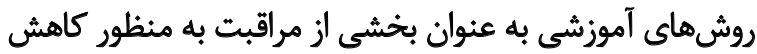

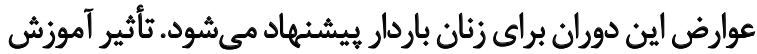

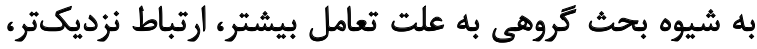

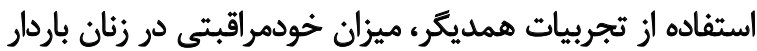

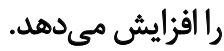

عدم همكارى و حضور نامرتب برخى از شركت إنندئان در

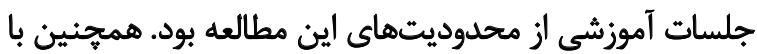

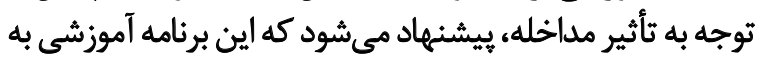
صورت رايكان توسط برسنل مراكز سلامت جامعه در اختيار زناني

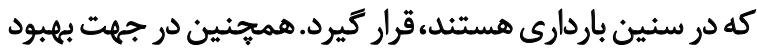

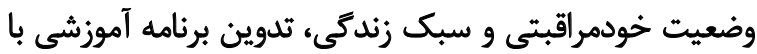

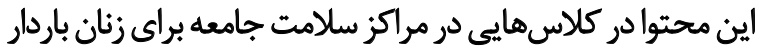

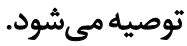

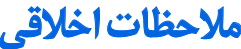

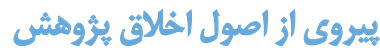

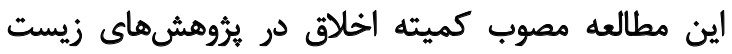

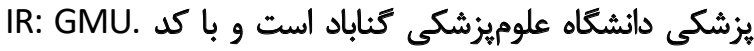

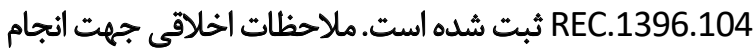

در مطالعه نصيرى آموزش به روش بركشتى بر خودمراقبتى

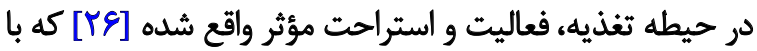

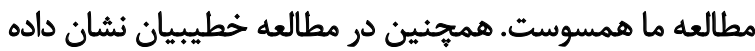

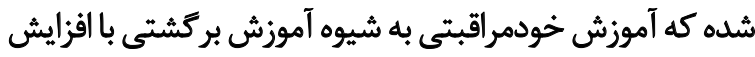

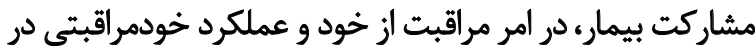

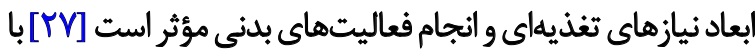

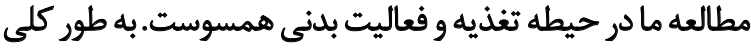

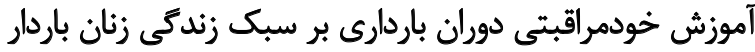

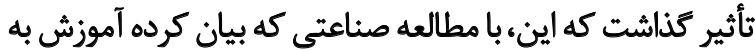

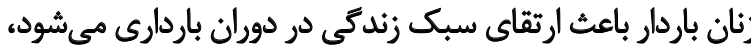

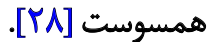

در ميانگين نمرات سبك زندگى بين سه كروه بعد از مداخله

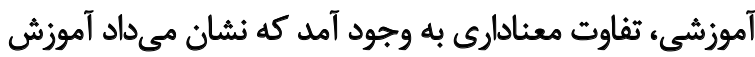

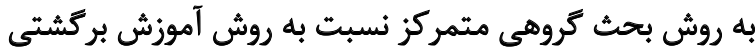

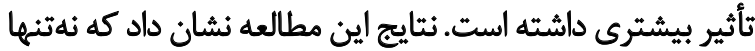

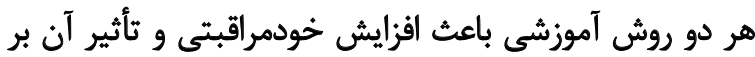

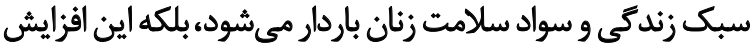

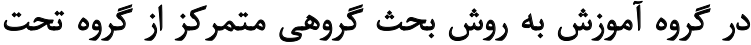

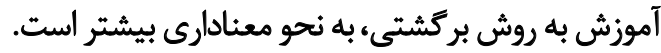

در خصوص مقايسه اين دو شيوه آموزشى در خودمراقبتى زنان

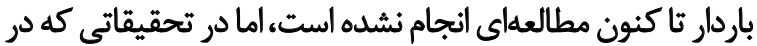

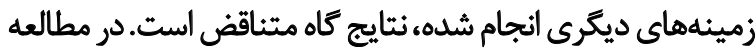

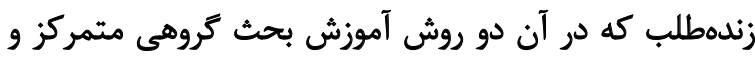

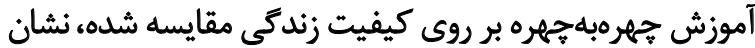

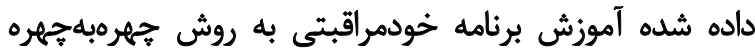

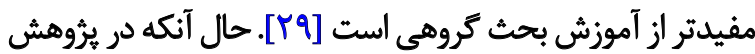

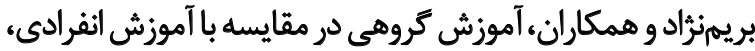

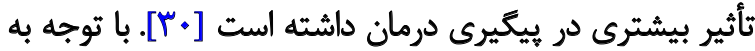

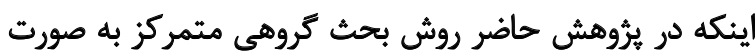

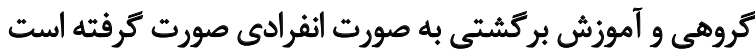
شايد بتواند با نتايج مطالعه ما همسو باشند.

اين تفاوتها مىتواند دلايل زيادى داشته باشد، از جمله

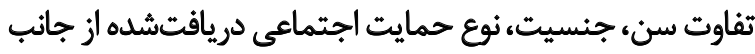

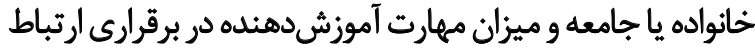

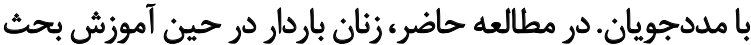

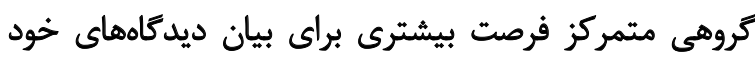

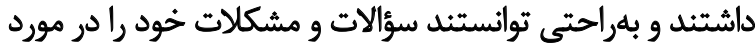

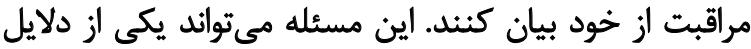

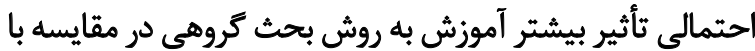

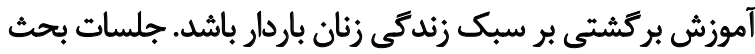

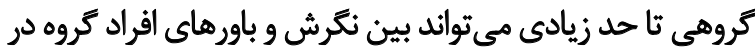

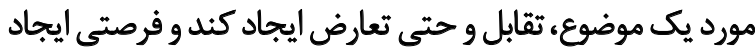

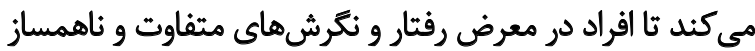


اين يرؤهش شامل هماهنغي هاي لازم و كسب اجازه براى ورود به

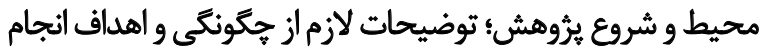

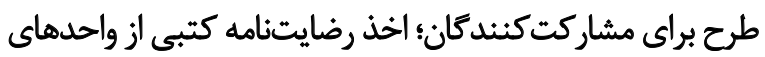

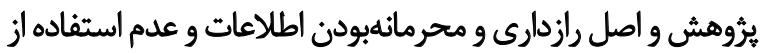
نام شركت كنيدًان بودي.

$$
\text { امامى مالى }
$$

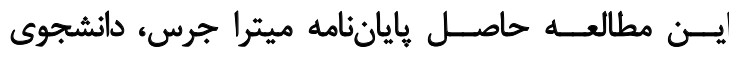

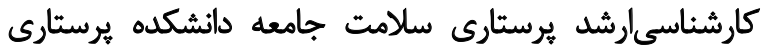

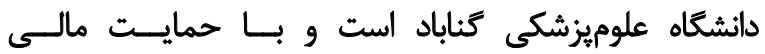

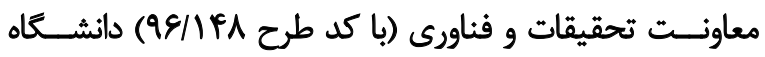

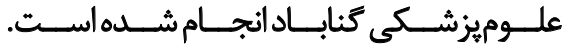

مشار كت ثويسئد مَّان

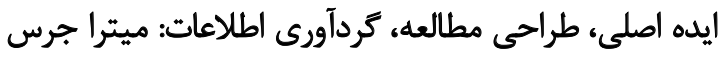

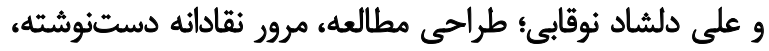

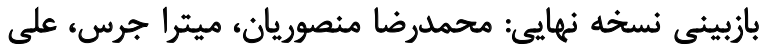
دلشاد نوقابي و حسين نظامي؛ آناليز دادهها؛ حسين نظامئ ميتر

$$
\text { تمارض مناقع }
$$

نويسندكان اين مقاله اعلام ميدارند كه هيجتونه تعارض منافعى در اين نوشتار وجود ندارد.

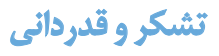

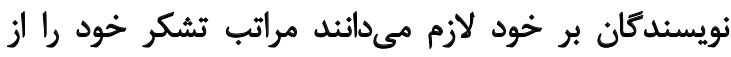

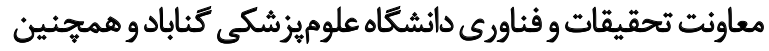

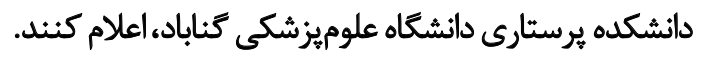




\section{References}

[1] Memari A, Ramim T, Amini M, Mehran A, Ajorloo A, Shakibaei P. [Investigation of effects of aerobic exercise on pregnancy and its circumstances (Persian)]. Journal of Hayat. 2006; 12(3):35-41. http://hayat. tums.ac.ir/article-1-201-en.html

[2] Sedaghati P, Ardjmand A, Sedaghati N. [Does regular ergometeric training have any effect on the pregnancy outcome? (Persian)]. Iranian Journal of Pediatrics. 2006; 16(3):325-31. https://tspace.library.utoronto.ca/ handle/1807/58307

[3] Moravcová $\mathrm{M}$, Mareš J, Ježek $\mathrm{S}$. Menopause Rating Scale-validation Czech version specific instrument for assessing health-related quality of life in postmenopausal women. Ošetřovatelství A Porodni Asistence. 2014; 5(1):36-45. https://www.researchgate.net/publication/269687724

[4] Olds SB. Maternal-newborn nursing \& women's health care. Upper Saddle River, NJ: Pearson Prentice Hall; 2004. https://books.google.com/ books?id=JMHjzAEACAAJ\&da

[5] Heaman MI, Newburn-Cook CV, Green CG, Elliott L, Helewa ME. Inadequate prenatal care and its association with adverse pregnancy outcomes: A comparison of indices. BMC Pregnancy and Childbirth. 2008; 8:15. [DOI:10.1186/1471-2393-8-15] [PMID] [PMCID]

[6] Wallace DA, Dodd MM, McNeil DA, Churchill AJ, Oelke ND, Arnold $S L$, et al. A pregnancy wellness guide to enhance care through self-assessment, personal reflection, and self. Journal of Obstetric, Gynecologic, and Neonatal Nursing. 2009; 38(2):134-47. [DOI:10.1111/j.15526909.2009.01008.x] [PMID]

[7] GHasemzadeh S, Dadmanesh M, Safari A, Ebrahimi S. [The Study on the knowledge, attitude and function of gestated mother's about gestational diabetes that referred to Army Khanvadeh Hospital from 2005 to 2006 (Persian)]. 2007; 5(3):1325-30. https://www.sid.ir/fa/journal/ ViewPaper.aspx?id=74778

[8] Derakhshan E, Shadzi Sh, Derakhshan F, Behjatian Z. [Effects of targeted education on knowledge and attitude of pregnant women regarding eclampsia (Persian)]. 2010; 6(3):443-9. http://hsr.mui.ac.ir/ index.php/jhsr/article/view/64

[9] Cunningham FG, Leveno KJ, Bloom SL, Spong CY, Dashe JS, Hoffman BL, et al. Williams obstetrics. New York: Mc Graw Hill Professional; 2014. https://www.worldcat.org/title/williams-obstetrics/oclc/892490452

[10] Mollakhalili H, Papi A, Zare-Farashbandi F, Sharifirad GR, Hasanzadeh A. A survey on health literacy of inpatient's educational hospitals of Isfahan University of Medical Sciences in 2012. Journal of Education and Health Promotion. 2014; 3:66. [DOI:10.4103/2277-9531.134804]

[11] Downey LVA, Zun LS. Assessing adult health literacy in urban healthcare settings. Journal of the National Medical Association. 2008; 100(11):1304-8. [DOI:10.1016/50027-9684(15)31509-1]

[12] Sajjadi H, Hosseinpour N, Sharifian Sani M, Mahmoodi Z. [Association between health literacy and life style in married rural women in Izeh, Iran (Persian)]. Journal of Health. 2016; 7(4):479-89. http://healthjournal.arums.ac.ir/article-1-1056-en.htm|

[13] Cassidy T. Stress, healthiness and health behaviours: An exploration of the role of life events, daily hassles, cognitive appraisal and the coping process. Counselling Psychology Quarterly. 2000; 13(3):293-311. [DOI:10.1080/09515070010028679]

[14] Bartlett EE. The contribution of school health education to community health promotion: What can we reasonably expect? American Journal of Public Health. 1981; 71(12):1384-91. [DOI:10.2105/ AJPH.71.12.1384] [PMID] [PMCID]
[15] Libbus MK, Sable MR. Prenatal education in a high-risk population The effect on birth outcomes. Birth. 1991; 18(2):78-82. [DOI:10.1111/ j.1523-536X.1991.tb00064.x] [PMID]

[16] Browner C, Preloran M, Press NA. The effects of ethnicity, education and an informational video on pregnant women's knowledge and decisions about a prenatal diagnostic screening test. Patient Education and Counseling. 1996; 27(2):135-46. [DOI:10.1016/0738-3991(95)00796-2]

[17] Azadbakht L, Haghighatdoost F, Esmaillzadeh A. [Comparing the effect of teaching based on problem solving method versus lecturing method regarding first diet therapy course for students of nutritional science (Persian)]. Iranian Journal of Medical Education. 2011; 10(5):1093-101. http://ijme.mui.ac.ir/article-1-1451-en.html

[18] Wong LP. Focus group discussion: A tool for health and medical research. Singapore Medical Journal. 2008; 49(3):256-60. [PMID]

[19] Baghcheghi N, Kouhestani HR, Rezaei K. [Comparison of the effect of teaching through lecture and group discussion on nursing students' communication skills with patients (Persian)]. Iranian Journal of Medical Education. 2010; 10(3):211-8. http://ijme.mui. ac.ir/article-1-1307-en.html

[20] Adib hajbagheri M, Parvizi S, Salsali M. [Qualitative research methods (Persian)] Tehran: Boshra Publisher; 2012. http://www.sciepub. com/reference/150995

[21] Ha Dinh TT, Bonner A, Clark R, Ramsbotham J, Hines S. The effectiveness of the teach-back method on adherence and self-management in health education for people with chronic disease: A systematic review. JBI Database of Systematic Reviews and Implementation Reports. 2016 14(1):210-47. [DOI:10.11124/jbisrir-2016-2296] [PMID]

[22] Wilson FL, Mayeta-Peart A, Parada-Webster L, Nordstrom C. Using the teach-back method to increase maternal immunization literacy among low-income pregnant women in Jamaica: A pilot study. Journal of Pediatric Nursing. 2012; 27(5):451-9. [DOI:10.1016/j. pedn.2011.05.004] [PMID]

[23] White M, Garbez R, Carroll M, Brinker E, Howie-Esquivel J. Is "teachback" associated with knowledge retention and hospital readmission in hospitalized heart failure patients? Journal of Cardiovascular Nursing. 2013; 28(2):137-46. [DOI:10.1097/JCN.0b013e31824987bd] [PMID]

[24] Reusch A, Ströbl V, Ellgring H, Faller H. Effectiveness of small-group interactive education vs. lecture-based information-only programs on motivation to change and lifestyle behaviours. A prospective controlled trial of rehabilitation inpatients. Patient Education and Counseling 2011; 82(2):186-92. [DOI:10.1016/j.pec.2010.04.031] [PMID]

[25] Shakeri M, Fekri Sh, Shahnavaz A, Shakibazadeh E. [Effectiveness of a group-based educational program on physical activity among pregnant women (Persian)]. Journal of Hayat. 2012; 18(3):1-9. http://hayat.tums. ac.ir/article-1-18-en.html

[26] Nasiri A, Poodineh Moghadam M. Effect of an educational plan based on teach-back method on hemodialysis patients' self-care deficits. Modern Care Journal. 2012; 9(4):344-54. https://www.semanticscholar. org/paper/Effect-of-an-educational-plan-based-on-Teach-Back-NasiriMoghadam/e8d7061b97602ee21133970e6225b6865939f9e6

[27] Oshvandi Kh, Jokar M, Khatiban M, Keyani J, Yousefzadeh MR, Sultanian AR. [The effect of self care education based on Teach Back method on promotion of self care behaviors in type II diabetic patients: A clinical trial study (Persian)]. Iranian Journal of Diabetes and Metabolism. 2014 13(2):131-44. http://ijdld.tums.ac.ir/article-1-5109-en.html

[28] Sanaati F, Mohammad Alizadeh S, Mirghafourvand M, Alizadeh Sharjabadi F, Galeshi M. [The effect of training pregnant women and their husbands on health promoting behaviors during pregnancy and post- 
partum period (Persian-English)]. Journal of Babol University of Medical Sciences. 2018; 20(5):38-47. http://jbums.org/article-1-7029-en.html

[29] Zendehtalab HR. [Comparing the effect of face to face and group discussion teaching methods on quality of life improvement among patients suffering from multiple sclerosis (Persian)]. Iranian Journal of Medical Education. 2013; 13(9):711-20. http://ijme.mui.ac.ir/article1-2595-fa.html

[30] Borimnejad L, Assemi S, Samiei N, Haghani H. [Comparison of group and individual training on the patients' compliance and incidence of warfarin side effects after cardiac valve replacement (Persian)]. Iranian Journal of Medical Education. 2012; 12(1):10-8. http://ijme.mui.ac.ir/ article-1-1721-fa.html

[31] Rahmati A, Etemadi A. [The effect of teaching coping skills on the-students attitudes towards drug abuse -using group discussion (Persian)]. Journal of Psychology. 2007; 1(4):117-41. https://www.sid.ir/fa/journal/ ViewPaper.aspx?id=87879

[32] Mahram M, Mahram B, Mousavinasab SN. [Comparison between the effect of teaching through student-based group discussion and lecture on learning in medical students (Persian)]. Strides in Development of Medical Education. 2009; 5(2):71-9. https://www.sid.ir/fa/journal/ ViewPaper.aspx?id $=89001$

[33] Khosravi Sh, Pazargadi M, Ashktorab T. [Nursing students` viewpoints on challenges of student assessment in clinical settings: A qualitative study (Persian)]. Iranian Journal of Medical Education. 2012; 11(7):73548. http://ijme.mui.ac.ir/article-1-1432-en.html

[34] Powell RA, Single HM. Focus groups. International Journal for Quality in Health Care. 1996; 8(5):499-504. [DOI:10.1093/intqhc/8.5.499]

[35] Boger EJ, Demain SH, Latter SM. Stroke self-management: A focus group study to identify the factors influencing self-management following stroke. International Journal of Nursing Studies. 2015; 52(1):175-87. [DOI:10.1016/j.ijnurstu.2014.05.006] [PMID]

[36] Millar B, Maggs C, Warner V, Whale Z. Creating consensus about nursing outcomes. I. An exploration of focus group methodology. Journal of Clinical Nursing. 1996; 5(3):193-7. [DOI:10.1111/j.1365-2702.1996. tb00249.x] [PMID]

[37] Brown MM, Mack KM, Guzzetta CE, Tefera E. The feasibility of using teach-back to reinforce discharge instructions and its influence on the number of 30-day readmissions of heart failure patients. Heart \& Lung: The Journal of Acute and Critical Care. 2014; 43(4):379. [DOI:10.1016/j. hrtlng.2014.06.004]

[38] Mohammadi Zeidi I, Pakpour Hajiagha A, Mohammadi Zeidi B. [Reliability and validity of Persian version of the health-promoting lifestyle profile (Persian)]. Journal of Mazandaran University of Medical Sciences. 2012; 21(1):102-13. http://jmums.mazums.ac.ir/article-1-955-en.html 